\title{
Evaluación de la influencia por variación del líquido de gobierno en el tiempo de esterilizado de conservas de anchoveta (Engraulis ringens) en envase 1/4 club
}

\author{
The influence generated in the heat treatment time by variation in liquid filling, in the production \\ of canned anchovy (Engraulis ringens) in format $1 / 4 \mathrm{club}$
}

1*Tito Eduardo Llerena Daza y ${ }^{2}$ Luís Enrique Tejada Atahualpa

\begin{abstract}
Resumen
Se determinó la influencia generada en el tiempo de esterilizado por la variación del líquido de cobertura en la producción de conservas de anchoveta en tipo entero de envase 1/4 Club RR-125. Estas comparaciones se realizaron mediante pruebas de penetración de calor comparativas para cada producto con su líquido de gobierno respectivo teniendo como consideración la similitud de los parámetros restantes; fijando como la letalidad objetivo $F_{0}=6$ min para alimentos de baja acidez. Se demostró que el comportamiento de la curva de penetración de calor para la conserva en salsa de tomate como líquido de coberturas es de tipo quebrada $\left(f_{h}=18,5 \mathrm{~min}\right.$ y $\left.f_{l}=36,5 \mathrm{~min}\right)$ y que el tiempo de procesamiento en envase $1 / 4$ Club es de $49 \mathrm{~min}$, para anchoveta en aceite vegetal $47 \min \left(f_{h}=22,5 \mathrm{~min}\right)$ y para anchoveta en agua y sal 40 $\min \left(f_{h}=17 \mathrm{~min}\right)$.
\end{abstract}

Palabras claves: líquido de gobierno, tiempo de esterilización, anchoveta.

\begin{abstract}
Has been determined the influence generated in the heat treatment time by variation in liquid filling, in the production of canned foods in this case being canned anchovy in the container $1 / 4$ Club RR-125. To determine whether the factor has a significantly influence in the time of sterilization, and proceeded to test heat penetration process by comparing different treatments for the factor with the other parameters in similar conditions; and finally get different time of sterilization with the object lethality for low-acid food, $F_{0}$ value $=6 \mathrm{~min}$ It was shown that the behavior of the heat penetration curve for canned with tomato sauce as a liquid type of coverage is broken $\left(f_{h}=18,5 \mathrm{~min}\right.$ and $\left.f_{l}=36,5 \mathrm{~min}\right)$ and the processing time in $1 / 4$ Club cane is $49 \mathrm{~min}$, in anchovies in vegetable oil for $47 \mathrm{~min}\left(f_{h}=22,5 \mathrm{~min}\right)$ and in anchovy in salted water $40 \mathrm{~min}$ $\left(f_{h}=17 \mathrm{~min}\right)$.
\end{abstract}

Key words: filling media, sterilization time, anchovy.

\section{Introducción}

Se conoce como tratamiento térmico a todos los procedimientos los cuales tienen como finalidad la destrucción de microorganismos mediante acción del calor (Casp-Vanaclocha y Abril-Requena, 2003). Para el caso de alimentos de baja acidez, el alimento se somete a una temperatura y presión de vapor suficientemente altas por un tiempo determinado como para que se consiga el efecto letal deseado, a esta operación se le conoce como esterilización (Karel and Lund, 2003). Se toma como referencia la destrucción de la bacteria "Clostridium botulinum" altamente letal y cuyas esporas son especialmente resistentes al calor (Perkins, 1964). Siempre y cuando se conserve la hermeticidad del producto se obtienen productos con vida útil muy prolongada (Hersom and Hulland, 1974).

La temperatura y tiempo de esterilización se dan en función de la velocidad de penetración del calor en el alimento, de las condiciones de calentamiento, el tamaño del envase, del estado físico del producto, entre otras (Berry and Pflug, 2003). Estas características sin lugar a dudas regulan las leyes físicas de transferencia de calor que permiten predecir el fenómeno de calentamiento y determinar las condiciones óptimas de operación (CaspVanaclocha y Abril-Requena, 2003).

El propósito del estudio de penetración de calor es el de determinar mediante ciclos simulados de esterilización, el comportamiento del calentamiento y enfriamiento de un producto y su envase en un sistema especifico de autoclave para establecer un proceso térmico seguro y evaluar sus desviaciones (Barbosa-Canova et al., 2000).

Debido a las características de producción que tiene cada empresa, los estudios que se llevan a cabo llegan a obtener distintos tiempos de esterilizado a una misma

\footnotetext{
${ }^{1}$ Departamento Académico de Acuicultura e Industria Pesquera, Facultad de Pesquería, Universidad Nacional Agraria La Molina, Apartado postal 12-056 - La Molina, Lima (Perú). Email: telld@lamolina.edu.pe.

*Autor de correspondencia.

${ }^{2}$ Egresado de la Facultad de Pesquería. Universidad Nacional Agraria La Molina, Lima.
} 
temperatura siendo el mismo producto y presentación el que se procesa. Esto sin lugar a duda hace ver que la velocidad de penetración de calor se encuentra también en función a factores productivos los cuales comúnmente en la práctica no se toman en cuenta, demostrando la necesidad de estas pruebas y así asegurar la esterilidad comercial del producto (Casp-Vanaclocha y AbrilRequena 2003).

En este trabajo se analizará la influencia generada por la variación del líquido de gobierno en el tiempo de esterilizado para una letalidad deseada en conserva de anchoveta (Engraulis ringens) en envase 1/4 Club RR-125, en formato novedoso en relación a los envases populares oval $1 \frac{2}{2}$ lb y $1 \mathrm{lb}$ tall (ITP, 2002).

\section{Materiales y métodos}

Los ensayos experimentales se llevaron a cabo en la planta de conservas de la empresa Consorcio Pesquero "El Ferrol S.A.C". ubicada en la cuidad de Chimbote y Ancash.

\section{Materiales y equipos}

\section{Materia Prima}

Se utilizó anchoveta (Engraulis ringens) fresca refrigerada, así como también ingredientes necesarios para la preparación del líquido de gobierno como pasta de tomate, sal doméstica, azúcar, carboximetilcelulosa, glutamato monosódico, aceite vegetal y agua potable.

Equipos e implementos

- Computador de Valor $\mathrm{F}_{0}$, marca ELLAB, modelo Val Suite, con capacidad de 16 canales de salida de control. Dieciséis termocuplas o sensores de temperatura tipo T (cobre-constatán). Autoclave tipo estacionarias, horizontal, discontinuas como medio de calentamiento vapor saturado. Computadora personal con el software ELLAB. Valsuite incluido el cual registra los datos obtenidos. Adaptadores o "pasamuros" para la inclusión de los sensores en las conservas. Adaptadores o Racors de 1 1/4, 1 1/2, y 2 pulgadas para entrada de los sensores en las autoclaves.

- Materiales varios para la ubicación del bulbo del sensor en el punto frío de la conserva como distanciadores y o-rings.

- Envases de hojalata 1/4 club RR-125, de forma rectangular y construcción del tipo dos piezas, versión de tapa de "fácil apertura" (Easy open).

\section{Metodología Experimental}

Descripción del procedimiento diseñado para realizar las pruebas de penetración de calor en anchoveta para envase $1 / 4$ Club

Las pruebas de penetración de calor fueron realizadas en paralelo a la producción. Cada parámetro fue determinado por las características propias de producción: grado de calidad de la materia prima, formulación del líquido de cobertura seleccionado (en salsa de tomate, en aceite vegetal y en agua y sal) y el sistema de tratamiento térmico de la empresa conservera. El procedimiento fue realizado como sigue:

Selección: la materia prima fue seleccionada biométricamente, con el fin de procesar anchovetas de tallas grandes con medidas de 14,8 $\pm 0,6 \mathrm{~cm}$ de longitud total y $24,8 \pm 1,8 \mathrm{~g}$ peso. Para luego ser sometidas a corte HGT ( $\sin$ cabeza, sin vísceras y sin cola) y ser sumergidas en salmuera fría (3 - 5\%).

Salmuerado: se realizó la inmersión de la anchoveta en salmuera saturada (26\%), durante 25 - 30 min, para proveer firmeza de la materia prima frente a la cocción.

Envasado: para esta operación se utilizó el envase 1/4 Club RR-125 de dimensiones $104 \times 59,8 \times 29,8 \mathrm{~mm}$ de dos piezas con tapa abre fácil. El acomodo del pescado se realizó de forma horizontal a la base de la lata, buscando los individuos de mayor tamaño; cuanto mayor sea su tamaño, mayor es la transferencia conductiva (Mendoza, 1993). Se siguió el procedimiento recomendado por Alegre et al., (2004); para el diseño del ensayo se evaluó los factores críticos asociados al producto (NTS $\mathrm{N}^{\circ}$ 071-MINSA/DIGESA- V.01, 2008), para tal fin, se buscó obtener pesos de los sólidos cocidos más un 10 por ciento adicional del peso, un nivel mayor a lo acostumbrado al producto estándar, para obtener el incremento de pesos para la prueba de penetración de calor, sin poner en riesgo la confiabilidad del proceso se esterilización del producto tratado utilizando tres líquidos de cobertura (Tabla 1), por lo que se le agregó cantidades mayores de anchoveta cruda.

Cocción: se realizó en un cocinador continuo por $25 \mathrm{~min}$ a $90{ }^{\circ} \mathrm{C}$.

Tabla 1. Incremento de pesos para pruebas de penetración de calor

\begin{tabular}{|c|c|c|c|c|c|c|}
\hline \multirow{3}{*}{$\begin{array}{l}\text { Diferencia de } \\
\text { pesos }\end{array}$} & \multicolumn{3}{|c|}{$\begin{array}{l}\text { Contenido de } \\
\text { sólido }(\mathrm{g})\end{array}$} & \multirow{3}{*}{$\begin{array}{c}\text { Agregado } \\
\text { de sólido } \\
\quad(\%)\end{array}$} & \multirow{3}{*}{$\begin{array}{l}\text { Contenido } \\
\text { líquido de } \\
\text { gobierno } \\
\text { (g) }\end{array}$} & \multirow{3}{*}{$\begin{array}{c}\text { Contenido } \\
\text { total } \\
\text { (g) }\end{array}$} \\
\hline & \multirow{2}{*}{\multicolumn{3}{|c|}{ Crudo $\frac{\text { Cocido }}{\text { Min. Max. }}$}} & & & \\
\hline & & & & & & \\
\hline $\begin{array}{l}\text { Producto } \\
\text { estándar }\end{array}$ & 120 & 88 & 92,5 & -- & $32,5-37,0$ & 125 \\
\hline $\begin{array}{c}\text { Tratamientos } \\
\qquad \mathrm{T}_{1}, \mathrm{~T}_{2}, \mathrm{~T}_{3}\end{array}$ & 135 & 98 & 102 & $8,9-13,3$ & $23.0-27,0$ & 125 \\
\hline
\end{tabular}

Enfriado: concluida la operación de cocinado, las latas extraídas fueron enfriadas a la intemperie por un tiempo prudencial hasta una temperatura menor a $40{ }^{\circ} \mathrm{C}$, esto debido a que las pruebas de penetración de calor deben realizarse a temperaturas inferiores a lo acostumbrado en producción (Weddig et al., 1995).

Adición de líquido de cobertura: se agregó tres tipos de líquido de gobierno, uno por tratamiento (Tabla 2), La adición se realizó a bajas temperaturas con la finalidad de evitar el incremento de temperatura a la conserva. 
Tabla 2. Líquido de gobierno utilizado para cada tratamiento

\begin{tabular}{|c|c|c|}
\hline Tratamiento & $\begin{array}{l}\text { Líquido de } \\
\text { gobierno }\end{array}$ & Producto \\
\hline $\mathrm{T}_{1}$ & $\begin{array}{l}\text { Salsa de } \\
\text { tomate }\end{array}$ & $\begin{array}{c}\text { Entero de anchoveta en } \\
\text { salsa de tomate }\end{array}$ \\
\hline $\mathrm{T}_{2}$ & $\begin{array}{l}\text { Aceite } \\
\text { vegetal }\end{array}$ & $\begin{array}{c}\text { Entero de anchoveta en } \\
\text { aceite vegetal }\end{array}$ \\
\hline $\mathrm{T}_{3}$ & Agua y sal & $\begin{array}{c}\text { Entero de anchoveta en } \\
\text { agua y sal }\end{array}$ \\
\hline
\end{tabular}

Sellado: los envases fueron sellados herméticamente con una cerradora automática de latas al vacío, sin pasar previamente por el túnel de vacío (exhaustor) para evitar el incremento de temperatura.

Colocación de termocuplas: el sensor de temperatura fue ubicado en el punto de calentamiento más lento el cual para este caso se encontro ubicado en el centro geométrico del producto.

Esterilizado: Los envases con termocupla fueron ubicados en las canastillas de la autoclave en forma horizontal en zonas preestablecidas con más lento calentamientoenfriamiento, para el registro de penetración de calor. Finalizada la carga se sometieron al proceso térmico, aplicando el programa por la empresa de acuerdo a las características propias de entrada de vapor para el cumplimiento del tiempo de venteo y de levante. El tiempo de tratamiento térmico para todos los tratamientos fue de $50 \mathrm{~min}$ a una temperatura de proceso de $116^{\circ} \mathrm{C}$.

Enfriado: el producto fue enfriado hasta temperaturas menores de $40{ }^{\circ} \mathrm{C}$, esto debido a que las pruebas de penetración de calor deben realizarse a temperaturas inferiores a lo acostumbrado en producción (Weddig et al., 2005).

\section{Determinación de valor de esterilización}

El valor de esterilización $F_{0}$ de los tratamientos térmicos fue determinado mediante el Método General propuesto por Bigelow and Esty (1920), utilizando la ecuación (1), integración de la velocidad letal $(L)$ con respecto al tiempo $(T)$.

$$
F_{0}=\int_{t=0}^{t} L_{x} d t=10^{\left(\frac{T-T_{\text {ref }}}{\mathrm{Z}}\right)}
$$

Pare resolver el término integral debe convertirse el tiempo real de calentamiento de un proceso, a una temperatura específica, ecuación (2), en el tiempo que se requeriría a $121,1{ }^{\circ} \mathrm{C}$ para lograr la misma destrucción de C. botulinum. Para tal fin, se utilizó el perfil de temperaturas del punto de más lento calentamientoenfriamiento de cada muestra analizada. La letalidad del proceso fue calculada utilizando un computador de Valor $F_{0}, \mathrm{ELLA}^{\circledR}$, que calculó automáticamente la superficie de integración pare el valor de $\mathrm{Z}=10^{\circ} \mathrm{C}$.

$$
L_{i}=1 / \log ^{-1}[(121-T) / 10]
$$

\section{Determinación de los parámetros de penetración de calor}

Para la determinación de los parámetros que describe las características de penetración de calor de los productos esterilizados se registraron los datos tiempo-temperatura del punto de calentamiento más frío determinado anteriormente. Con los datos obtenidos, se graficaron las curvas de calentamiento y enfriamiento, a partir de las cuales se calculó la diferencia, $T_{1}-T_{t}$ (temperatura de procesamiento - temperatura del alimento) y se representaron con respecto al tiempo sobre papel semilogaritmico en cualquier tiempo t determinando $f_{h}$ (la inversa de la pendiente de la curva de calentamiento), $f_{c}$ (la inversa de la pendiente de la curva de enfriamiento), $T_{p s i h}$ (la temperatura pseudoinicial de calentamiento), $T_{p s i c}$ (la temperatura pseudoinicial de enfriamiento), $j_{h}$ (el factor de retraso en el calentamiento) y $j_{c}$ (el factor de retraso en el enfriamiento).

\section{Determinación del tiempo de tratamiento térmico}

El Valor $F_{o}$ (efecto letal) aplicado fue de un valor mínimo de 6 min (NTS 071-2008 MINSA). El producto fue tratado térmicamente a $116{ }^{\circ} \mathrm{C}$, registrándose el tiempo-temperatura del punto de calentamiento más lento determinado previamente. Con los parámetros de la cinética de destrucción del microorganismo de referencia $\left(D_{121,1}=0,21 \mathrm{~min}\right.$ y $\left.Z=10^{\circ} \mathrm{C}\right)$, los parámetros determinados de la curva de penetración de calor $\left(f_{h}, f_{c^{\prime}}\right.$ $\left.j_{h}, T_{p s i h}\right)$, y la gráfica $f / U$ : $\log \mathrm{g}\left({ }^{\circ} \mathrm{C}\right)$ para $\mathrm{m}+\mathrm{g}=100{ }^{\circ} \mathrm{C}$, se calculó el tiempo de tratamiento térmico para cada tratamiento, empleando el método de Ball mejorado (Paredes, 2007).

\section{Resultados y discusión}

\section{Características de los procesos térmicos}

Según los resultados de la Tabla 3 se puede apreciar que factores que pueden influir en el tiempo de esterilizado como la temperatura inicial, venteo y tiempo de levante o de puesta en régimen son similares para los tres casos, evitando alteraciones posibles en el valor $F_{0}$ final del producto.

Las diferencias observadas de uno o dos grados en la temperatura inicial, en el venteo o en el tiempo de levante se muestran de manera no influyentes en comparación al factor analizado debido a que no muestran relación con el valor $F_{0}$ De acuerdo a Sharma et al., (2003), el tiempo de muerte térmica en min es una función exponencial de la temperatura en ${ }^{\circ} \mathrm{C}$ ó ${ }^{\circ} \mathrm{F}$, así para el rango de grados de temperatura está trazado en escala aritmética, para el efecto letal el tiempo esta trazado en escala logarítmica. Por lo tanto, se demuestra que las variaciones del valor $F_{0}$ está relacionado con el líquido de gobierno como el factor crítico asociado al producto. 
Tabla 3. Características técnicas de las pruebas de penetración de calor realizadas para cada tipo de producto evaluado según el líquido de gobierno

\begin{tabular}{|c|c|c|c|}
\hline Tratamiento & $\mathrm{T}_{1}$ & $\mathrm{~T}_{2}$ & $\mathrm{~T}_{3}$ \\
\hline Líquido de gobierno & $\begin{array}{l}\text { Salsa } \\
\text { tomat }\end{array}$ & $\begin{array}{l}\text { Aceite } \\
\text { vegetal }\end{array}$ & $\begin{array}{c}\text { Agua y } \\
\text { sal }\end{array}$ \\
\hline $\begin{array}{l}\text { Temperatura } \mathrm{i} \\
\text { máxima del pr. }\end{array}$ & $25,39^{\circ} \mathrm{C}$ & $23,92^{\circ} \mathrm{C}$ & $24,53^{\circ} \mathrm{C}$ \\
\hline $\begin{array}{l}\text { Tem } 1 \\
\text { mínin }\end{array}$ & $21,11^{\circ} \mathrm{C}$ & $22,83{ }^{\circ} \mathrm{C}$ & $23,06^{\circ} \mathrm{C}$ \\
\hline Vent & $\begin{array}{l}10 \mathrm{~min}, \\
\text { hasta } \\
103,88^{\circ} \mathrm{C}\end{array}$ & $\begin{array}{l}11 \mathrm{~min} \\
\text { hasta } \\
104,32^{\circ} \mathrm{C}\end{array}$ & $\begin{array}{c}11 \mathrm{~min}, \\
\text { hasta } \\
103,59^{\circ} \mathrm{C}\end{array}$ \\
\hline $\begin{array}{l}\text { Tiempo de levante } \\
\text { (CUT) }\end{array}$ & $13 \mathrm{~min}$ & $14 \mathrm{~min}$ & $13 \mathrm{~min}$ \\
\hline $\begin{array}{c}\text { Temperatura del } \\
\text { proceso térmico }\left(T_{p}\right)\end{array}$ & $115,73^{\circ} \mathrm{C}$ & $115,74^{\circ} \mathrm{C}$ & $115,87^{\circ} \mathrm{C}$ \\
\hline $\begin{array}{l}\text { Tiempo del proceso } \\
\text { térmico }\left(t_{p}\right)\end{array}$ & $50 \mathrm{~min}$ & $50 \mathrm{~min}$ & $50 \mathrm{~min}$ \\
\hline$F_{o}$ menor y termopar & $\begin{array}{l}6,260 \min \\
\left(\mathrm{T}_{\mathrm{e} 10}\right)\end{array}$ & $\begin{array}{l}\text { 6,994 min } \\
\left(\mathrm{T}_{\mathrm{e} 6}\right)\end{array}$ & $\begin{array}{l}9,011 \mathrm{~min} \\
\left(\mathrm{~T}_{\mathrm{e} 5}\right)\end{array}$ \\
\hline$F_{o}$ promedio & $6,937 \mathrm{~min}$ & $8,400 \mathrm{~min}$ & $9,575 \mathrm{~min}$ \\
\hline$F_{o}$ mayor y termopar & $\begin{array}{c}\text { 7,379 min } \\
\left(T_{\mathrm{e} 7}\right)\end{array}$ & $\begin{array}{c}10,250 \mathrm{~min} \\
\left(\mathrm{~T}_{\mathrm{e} 11}\right)\end{array}$ & $\begin{array}{c}19,965 \mathrm{~min} \\
\left(\mathrm{~T}_{\mathrm{e} 14}\right)\end{array}$ \\
\hline
\end{tabular}

\section{Características de la curva de penetración de calor en los productos evaluados según el líquido de gobierno}

Mediante el método de Ball se determinaron los datos de penetración de calor, se procedió a graficar las curvas de calentamiento para los tres productos, las mismas que aparecen en la Figura 1 y de las que se obtuvieron los parámetros mostrados en la Tabla 4.

Tabla 4. Parámetros de la curva de penetración de calor según el método de Ball para cada tipo de líquido de gobierno

\begin{tabular}{cccc}
\hline \multirow{2}{*}{ Parámetros } & \multicolumn{3}{c}{ Producto: anchoveta entera en } \\
\cline { 2 - 4 } & salsa de tomate aceite vegetal & agua y sal \\
\hline$f_{h}$ & $18,5 \mathrm{~min}$ & $22,5 \mathrm{~min}$ & $17,0 \mathrm{~min}$ \\
$f_{1}$ & $44,0 \mathrm{~min}$ & -- & -- \\
$j_{h}$ & 0,877 & 0,822 & 0,840 \\
$T_{p s i h}$ & $83^{\circ} \mathrm{C}$ & $75^{\circ} \mathrm{C}$ & $78^{\circ} \mathrm{C}$ \\
\hline
\end{tabular}

Donde: $f_{h}$ es la inversa negativa de la pendiente de la curva de calentamiento; $f_{l}$ es la inversa negativa de la pendiente de la curva de calentamiento quebrada; $j_{h}$ es el factor de retraso en la curva de calentamiento, obtenida del cociente: $\left(T_{p s i h}-T_{\mathrm{o}}\right) /\left(T_{p}-T_{0}\right) ; T_{p s i h}$ es la temperatura pseudoinicial de calentamiento.

Las curvas de penetración de calor en coordenadas logarítmica-lineal para cada tipo de líquido de gobierno nos muestran que, tanto la temperatura seudoinicial $\left(T_{p s i h}\right)$, y el factor lag de calentamiento $\left(j_{h}\right)$ se asemejan; por lo que se puede decir que el incremento o levante de temperatura por producto del calor en los tres productos fueron similares.

Rochabrun (1996) menciona que cuando mayor sea el valor del factor lag, indica que existe un retraso en la elevación de la curva. Caso similar ocurre para los valores calculados para los factores lag de calentamiento o factor de retraso en el enlatado de anchoveta en salsa de tomate $\left(j_{h}=0,877\right)$, en aceite vegetal $\left(j_{h}=0,822\right)$ y en agua y sal $\left(j_{h}=0,840\right)$, que fueron bajos como es en este caso.

La diferencia significativa de las curvas de penetración calor se muestra en el tiempo, el cual la temperatura recorre un ciclo logarítmico $\left(f_{h}\right)$. Las curvas de los productos que tienen como líquido de gobierno salsa de tomate y salmuera muestran un valor $f_{h}$ menor lo que quiere decir que inicialmente se calentaron con mayor rapidez que la conserva con aceite como líquido de cobertura.

El aceite vegetal a pesar poseer de tener un menor calor específico $\left(c_{p}: 0,478 \mathrm{cal} / \mathrm{g} .{ }^{\circ} \mathrm{C}\right)$ que la salmuera $\left(c_{p}: 0,990\right.$ $-0,805 \mathrm{cal} / \mathrm{g} .{ }^{\circ} \mathrm{C}$ ), es muy posible que la viscosidad que posee este líquido al ser mayor no permita en los inicios del procesamiento térmico una distribución completa del calor en la conserva. Por lo que tomando lo expresado por Mendoza (2003) y Casp-Vanaclocha y AbrilRequena (2003), las corrientes convectivas se pueden ver interrumpidas, alcanzando rápidamente temperaturas altas en las zonas externas más no en el punto de calentamiento más lento de la conserva. Un caso parecido debe ocurrir con la salsa de tomate ya que contiene agua en su composición y no es completamente homogénea.

Sin embargo la curva de penetración de calor de enlatado de anchoveta en salsa de tomate es del tipo de curva quebrada de la cual se obtuvo el valor $f_{1}$ de $44 \mathrm{~min}$, Esta segunda inclinación de la curva determina finalmente que el valor letal de esta conserva sea el menor para esta evaluación.

Stumbo (1973) señala que los productos más comunes que exhiben curvas de calentamiento quebradas son los productos que se calientan durante un tiempo por convección, y luego, debido al almidón o a algún otro agente espesante, se calientan por conducción.

\section{Comparación de los valores letales obtenidos mediante las pruebas de penetración de calor}

La conserva a la cual se agregó agua y sal como líquido de gobierno obtuvo el mayor promedio letal (9,6 min), en comparación a la que se agregaron aceite $(8,2 \mathrm{~min})$ y salsa de tomate $(6,9 \mathrm{~min})$ mostrándose una diferencia estadísticamente significativa $(\mathrm{p}<0,05)$.

Se aprecia en la Tabla 5, que la dispersión del valor promedio o desviación estándar al igual que el rango letal es mayor para las conservas adicionadas con aceite vegetal como líquido de gobierno. Esto se puede deber a la diferencia del tamaño de las piezas sólidas, esto ocasionaría que un incremento de la conductividad y por lo tanto es más tardío la penetración de calor (Mendoza, 2003). 


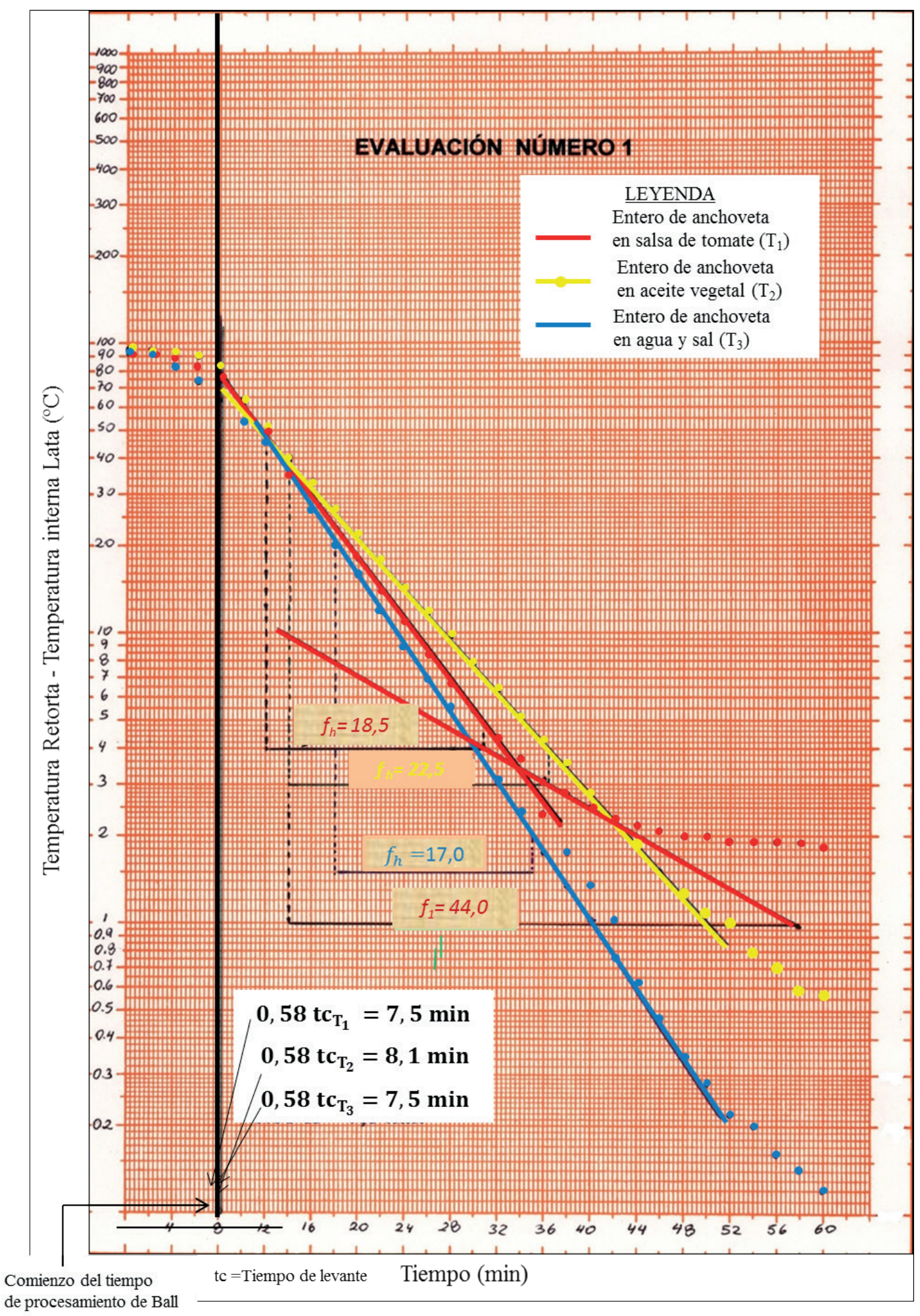

Figura 1. Gráfica determinada por el método de Ball para las conservas de anchoveta evaluadas según el líquido de gobierno 


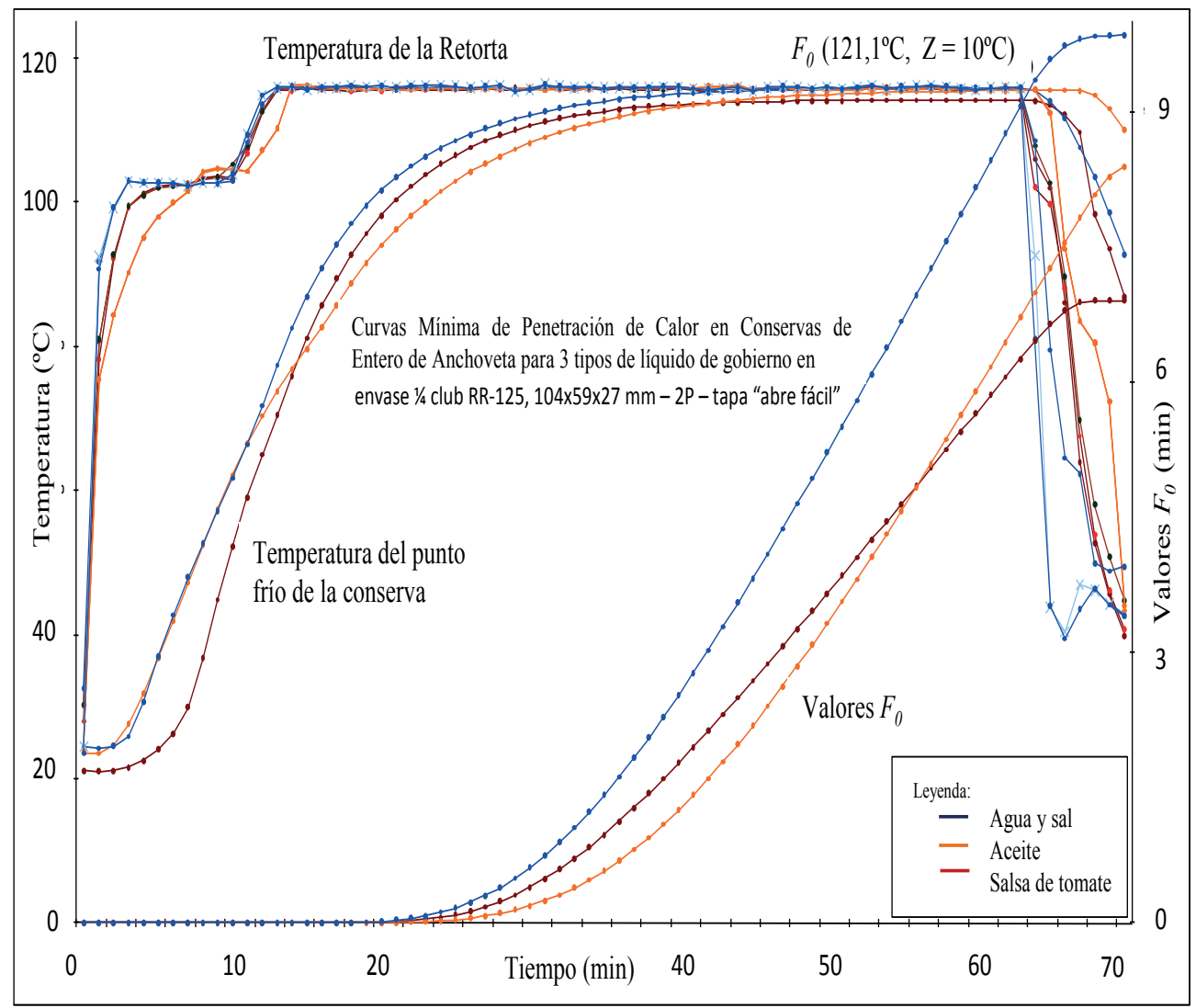

Figura 2. Comparación de las curvas de penetración de calor co menor letalidades para la evaluación de distintos tipos de líquido de gobierno

En la Figura 2 se aprecian de manera superpuesta las curvas con menor letalidad para cada tratamiento. Los productos sometidos a los tratamientos térmicos, cumplieron con las condiciones de esterilidad comercial, valor Fo mínimo de $6 \mathrm{~min}$, de acuerdo a la NTS No 071 (MINSA/DIGESA, 2008).

Tabla 5. Valores letales según el líquido de gobierno, obtenidos para cada termocupla

\begin{tabular}{cccc}
\hline Tratamiento & $\mathrm{T}_{1}$ & $\mathrm{~T}_{2}$ & $\mathrm{~T}_{3}$ \\
\hline Tipo de líquido & $\begin{array}{c}\text { Salsa de } \\
\text { tomate }\end{array}$ & $\begin{array}{c}\text { Aceite } \\
\text { vegetal }\end{array}$ & Agua y sal \\
termopar 1 & 7,311 & 6,994 & 9,011 \\
termopar 2 & 7,353 & 8,136 & 9,661 \\
termopar 3 & 7,379 & 9,479 & 9,455 \\
termopar 4 & 6,933 & 7,611 & 9,892 \\
termopar 5 & 6,728 & 7,666 & 9,814 \\
termopar 6 & 6,260 & 9,750 & 9,527 \\
termopar 7 & 6,813 & 8,232 & 9,619 \\
termopar 8 & 7,154 & 7,725 & 9,588 \\
termopar 9 & 7,337 & 8,256 & 9,058 \\
termopar 10 & 6,607 & 7,938 & 9,965 \\
termopar 11 & 6,743 & 8,167 & 9,854 \\
termopar 12 & $\mathbf{6 , 6 3 7}$ & $\mathbf{8 , 8 3 1}$ & $\mathbf{9 , 4 5 1}$ \\
Valores mínimos & 6,260 & 6,994 & 9,011 \\
SD & 0,370 & 0,801 & 0,316 \\
Promedio & 6,965 & 8,178 & 9,586 \\
Rango letal & 1,119 & 2,756 & 0,954 \\
\hline
\end{tabular}

Determinación del tiempo de esterilizado para los diferentes tratamientos de la evaluación número uno

Registradas las temperaturas y calculado los valores letales mediante el método de Ball mejorado (Paredes, 2007) por el programa Ellab - valsuite se determinó los valores de tiempos de procesamiento requerido para el proceso (Tabla 6), considerando que se debe cumplir el valor de esterilidad comercial para cada tratamiento.

De acuerdo a lo estipulado por el Instituto Tecnológico Pesquero de $6 \mathrm{~min}$ como valor letal mínimo de esterilización (ITP, 2005), el tiempo de proceso para la conserva de anchoveta en salsa de tomate es de $49 \mathrm{~min}$ como mínimo, para anchoveta en aceite vegetal que es de 47 min y para la conserva de anchoveta en agua y sal que es de $40 \mathrm{~min}$.

De acuerdo a lo mostrado por el cuadro presentado el tiempo de esterilizado es influido por el líquido de gobierno a utilizar. 
Tabla 6. Tiempo de proceso y valores letales cercanos a 6 min para el producto entero de anchoveta según el líquido de gobierno.

\begin{tabular}{cccccc}
\hline \multicolumn{2}{c}{ Prueba 1 } & Prueba 2 & \multicolumn{2}{c}{ Prueba 3 } \\
\hline $\begin{array}{c}\mathrm{T}_{1} \text { : anchoveta entera } \\
\text { en salsa de tomate }\end{array}$ & $\begin{array}{c}\mathrm{T}_{2} \text { : anchoveta } \\
\text { entera en aceite } \\
\text { vegetal }\end{array}$ & $\begin{array}{c}\mathrm{T}_{3} \text { : anchoveta } \\
\text { entera en agua } \\
\text { y sal }\end{array}$ \\
\hline \multicolumn{2}{c}{ Termopar 10 } & \multicolumn{2}{c}{ Termopar 5 } & \multicolumn{2}{c}{ Termopar 5 } \\
\hline $\begin{array}{c}t_{p} \\
(\text { min) }\end{array}$ & $\begin{array}{c}\text { Valor } F_{0} \\
(\text { min) }\end{array}$ & $\begin{array}{c}t_{p} \\
(\text { min) }\end{array}$ & $\begin{array}{c}\text { Valor } F_{0} \\
(\text { min) }\end{array}$ & $\begin{array}{c}t_{p} \\
(\text { min) }\end{array}$ & $\begin{array}{c}\text { Valor } F_{0} \\
(\text { min })\end{array}$ \\
\hline 47 & 5,654 & 45 & 5,631 & 38 & 5,433 \\
48 & 5,856 & 46 & 5,901 & 39 & 5,726 \\
49 & 6,058 & 47 & 6,172 & 40 & 6,019 \\
50 & 6,260 & 48 & 6,445 & 41 & 6,314 \\
\hline
\end{tabular}

\section{Conclusiones}

La conserva de entero de anchoveta en salsa de tomate se muestra del tipo quebrada $\left(f_{h}=18,5 \min\right.$ y $f_{1}=36,5$ min) producto de la composición que posee por lo que necesita un mayor tiempo de esterilizado $\left(t_{p}=49 \mathrm{~min}\right)$ que las conservas en aceite vegetal $\left(t_{p}=47 \mathrm{~min}\right)$ y agua y sal $\left(t_{p}=40 \mathrm{~min}\right)$, las cuales muestran curvas de penetración de calor con una sola pendiente $\left(f_{h}=22,5\right.$ min y $f_{h}=17$ min respectivamente). Para un valor $F$ requerido $\left(F_{0}\right.$ $=6 \mathrm{~min}$ ), el líquido de cobertura de la conserva es el factor que influye de manera significativa en el tiempo de procesamiento térmico de esterilizado. Esto debido a que las características propias de cada líquido como viscosidad, capacidad calorífica o composición del mismo determinan la transferencia convectiva del calor en la conserva, la cual es la transferencia de calor del tipo más eficiente como lo indica Vanaclocha y Requena (1999). Esto se demostró de acuerdo a las diferencias de las curvas de penetración obtenidas

\section{Literatura citada}

Alegre, C., Mac Cotrina, G. y Leyton, M. 2004. Guía de prácticas para la aplicación de los principios generales del procesamiento térmico en la industria conservera. Instituto Tecnológico Pesquero del Perú (ITP). Lima, Perú.

Barbosa-Canovas, G.V., Ma, L. y Barletta, B. 2000. Manual de Laboratorio de ingeniería de los alimentos. Editorial Acribia. Zaragoza, España.

Berry M. R., and Pflug I. J. 2003. Canning Principles. In: Encyclopedia of Food Sciences and Nutrition.

Bigelow, W., and Esty, J. 1920. The Thermal death point in relation to time of typical thermophilic organisms. The Journal of Infectious Diseases, 27(6), 602-617. Recuperado de http://jid.oxfordjournals.org/ content/27/6/602.full.pdf + html

Casp-Vanaclocha, A. y Abril-Requena, J. 2003. Procesos de conservación de alimentos. 2a. ed. Ediciones Mundi-Prensa. Madrid, España.
Hersom, A. C., and Hulland, E. D. 1974. Canned foods: An introduction to their microbiology. Chem. Publ. Co., New York, USA.

Instituto Tecnológico Pesquero del Perú (ITP). 2002. Investigación y desarrollo de productos pesqueros. Recuperado de https://es.slideshare.net/viplasmi/ proyectos-itp

Instituto Tecnológico Pesquero del Perú (ITP). 2005. Protocolo para el procesamiento de anchoveta en conservas. Lima, Perú.

Karel, M., and Lund, DB. 2003. Physical Principles of Food Preservation. New York: Marcel Dekker. 602 pp. 2nd ed.Recuperado de https://thunderbooks.files.wordpress. com/2009/05/physical-principles-of-preservation. pdf

Mendoza, F. 2003. Evaluación y optimización del tratamiento térmico en una crema base de olluco (Ullucus tuberosus) enlatada (Tesis para optar el título de Ingeniero en Industrias Alimentarias). Universidad Nacional Agraria La Molina (UNALM). Lima, Perú.

NTS 071-MINSA/DIGESA-V-01, 2008 Norma Sanitaria que establece los criterios microbiológicos de calidad sanitaria e inocuidad para los alimentos y bebidas de consumo humano. Lima, Perú.

Rochabrun, J. R. 1996. Curso Internacional Tecnología de Procesamiento de Productos Pesquero. Separata. Curso Internacional. JICA/ITP. Perú.

Paredes, P. 2007. Introducción al tratamiento térmico en conservas. Instituto Tecnológico Pesquero del Perú (ITP). Lima, Perú.

Perkins, W. E. 1964. Prevention of botulism by thermal processing. In K. H. Lewis and K. C. Cassel, Jr. (editors), Botulism, p. 187-201. Public Health Servo Publ. 999- FPI, Public Health Serv., Cincinnati, Ohio. Recuperado de http://spo.nmfs.noaa.gov/mfr452/mfr4521.pdf

Perry, H. R., \&"Green, D. 2000. Perry's Chemical Engineers Handbook", Estados Unidos: McGraw-Hill, 2007.

Sharma, S. K., Mulvaney, S. J., Rizvi, S, H, 2003. Ingeniería de Alimentos. Operaciones unitarias y practices de laboratorio. Ed LIMUSA. S.A. México.

Stumbo, C. R. 1973. Thermobacteriology in food Procesing. Academic Press, New York.

Weddig, L.M., Balestrini, C. G., Shafer, B. D. 1995. Canned Foods: Principles of Thermal Process Control, Acidification and Container Closure Evaluation, sixth ed., The Food Processors Institute, Washington, DC. 